The Astrophysical Journal, 317:L13-L14, 1987 June 1

( 1987 . The American Astronomical Society. All rights reserved. Printed in U.S.A.

\title{
DISCOVERY OF THREE OBSCURED GLOBULAR CLUSTERS
}

\author{
S. DJORGOVSKI ${ }^{1,2}$ \\ Harvard-Smithsonian Center for Astrophysics \\ Received 1986 December 26; accepted 1987 March 12
}

\begin{abstract}
I report discovery of three previously uncataloged globular clusters $\mathrm{n}$ a direction of the Galactic Bulge. The clusters appear under a heavy foreground and extinction and are probably located close to the Galactic center. They are not associated with any known X-ray, $\gamma$-ray, or IR sources, including the IRAS point sources. One of the clusters may have a postcollapse core.
\end{abstract}

Subject headings: clusters: globular - galaxies: The Galaxy

Globular clusters are among the most stimulating and versatile astrophysical objects. They are the main testing ground for the dynamics of star clusters, a vigorous field with many new developments (cf. the review by Elson, Hut, and Inagaki 1987). They are also probes of the mass distribution and dynamics (and even the formation?) of our Galaxy and provide the benchmarks for the stellar evolution theory. There are about 130 Galactic globular clusters currently known, and it is estimated that up to several tens of clusters are still hidden in the obscured areas of the Bulge and the disk, the regions of particular importance for the theories of their dynamical evolution. Locating some of the "missing" clusters is thus a promising and potentially rewarding task.

Webbink (1985) provides an up-to-date list of known or suspected globular clusters; however, not all of the objects listed there are confirmed globulars as yet. Since the work of Terzan (1971 $a, b$; see also King 1972) which yielded a dozen new clusters, several obscured clusters were found: BH 229 (van den Bergh and Hagen 1975), Liller 1 (Liller 1977), and UKS 1 (Malkan, Kleinmann, and Apt 1980). Two possible clusters, Grindlay 1 (Grindlay and Hertz 1981) and Kodaira 1 (Kodaira 1983), were indicated through association with X-ray and IR sources, but visual/IR images of these objects still have not been published. Terzan, Bernard, and Ju (1978), Terzan and Ju (1980), and Terzan (1985) identified 41 "diffuse objects" on Schmidt plates of the Sagittarius cloud B, but none of them are as yet confirmed globular clusters, and some are clearly background galaxies or planetary nebulae. Several other objects listed by Webbink (1985) need a confirmation.

As a part of a project to look for possible obscured globular clusters, selected from the IRAS Point Source Cata$\log$ (Djorgovski 1987b, and in preparation), SRC Southern Sky survey $I$ band films were examined. The preliminary examination of the IRAS cluster candidate fields was done by eye, and with a hand-held magnifier. Most of the IRAS candidates are in "blank fields," and need a follow-up CCD imaging. In the course of this work, it was decided that not

\footnotetext{
${ }^{1}$ Visiting Astronomer, Cerro Tololo Interamerican Observatory, NOAO, operated by AURA, Inc., under contract with the NSF.

${ }^{2}$ Society of Fellows, Harvard University.
}

only the selected IRAS error circles will be examined, but rather the full Sky Survey films that were used for this purpose. The IRAS search fields covered so far have the area of over $100 \mathrm{~s} \mathrm{deg}^{2}$, and the extended search (the IRAS fields plus some of the surrounding area) perhaps about $150 \mathrm{~s} \mathrm{deg}^{2}$ (the exact number is hard to estimate, because of some redundancy overlap between the adjacent fields). This is how the new clusters reported here were found. One or two other, less probable cluster candidates still need confirming observations.

However, the faintness of these newly found objects, and the heavy foreground prevented their clear identification as globular clusters. It was necessary to get better imaging data. Several tens of CCD images of the three tentative cluster fields were obtained with the CTIO $1.5 \mathrm{~m}$ telescope, on the nights of UT 1986 September 13 and 15, in variable conditions. The detector was a TI $800 \times 800$ pixels, backcharged, low readout noise CCD, mounted at the Cassegrain (f/7.5) focus. Since the seeing was rather mediocre (FWHM $\sim 2^{\prime \prime}$ or worse), the device was read out in the $2 \times 2$ pixels binning mode, giving the effective pixel size of 0.545 , and the field size of $\sim 3.6$ arcmin. $^{2}$ The exposures were obtained in the $V R I$ bands and lasted from 1 to 10 minutes, depending on the bandpass, a presence of bright foreground stars, and the intensity of moonlight.

The data were processed with the standard, on-line reduction software. First, the bias level was determined from an overscan in each row, and subtracted (this removes the rowto-row pattern, and the DC offset), and then the average bias frames were subtracted from the data (this removes the column-to-column pattern). The data frames were then divided by the out-of-focus dome flatfields, which is an adequate procedure for the present purpose. Selected individual exposures are shown in Figure 1 (Plate L1).

All three of the cluster candidates were confirmed as globular clusters. The morphological criterion is that the object must be diffuse, centrally concentrated, of a circular shape, and with at least marginally resolved red giants (for example, background galaxies would be generally smaller, more elliptical, and unresolved). Images of several of the known Bulge clusters, obtained on the same nights, provide convenient 


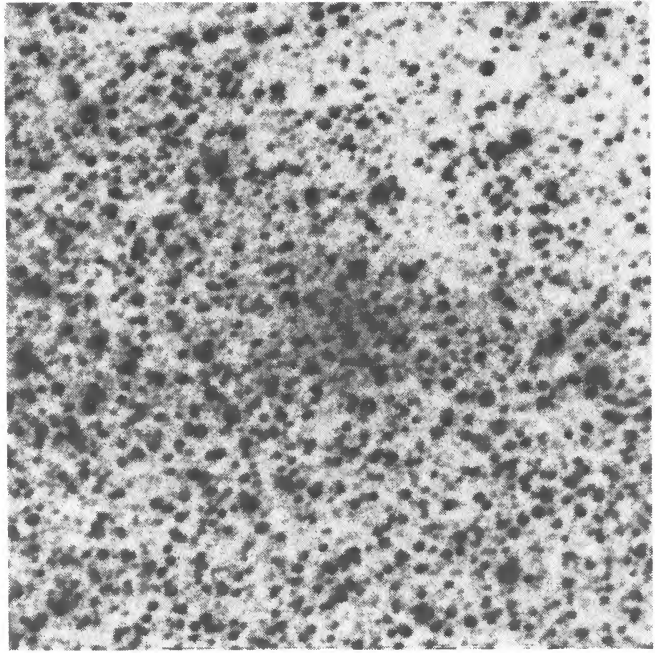

$\mathrm{R}$

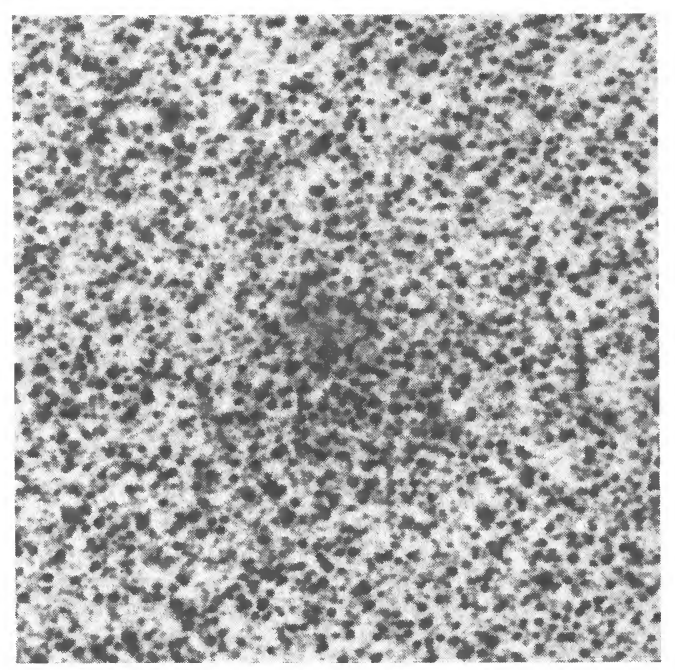

$\mathrm{R}$

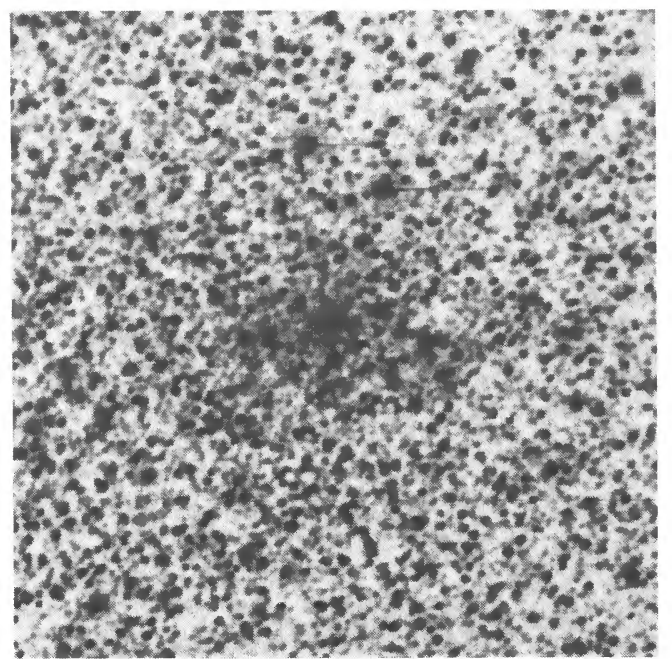

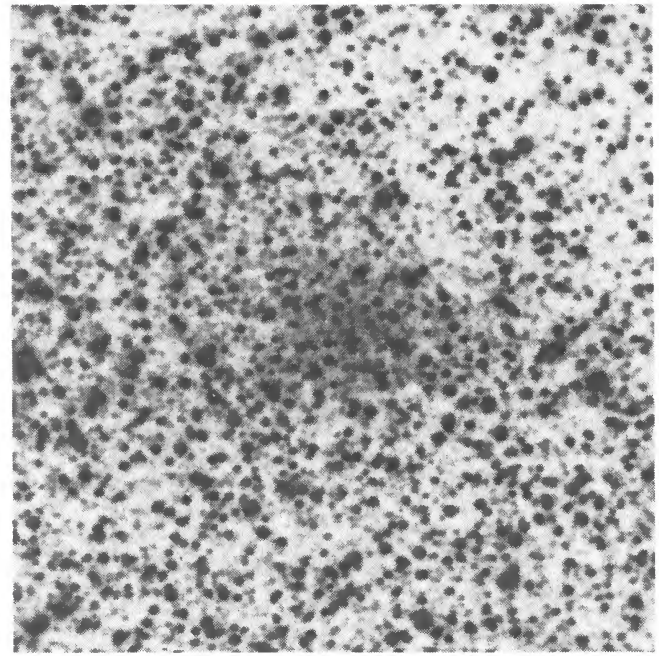

I
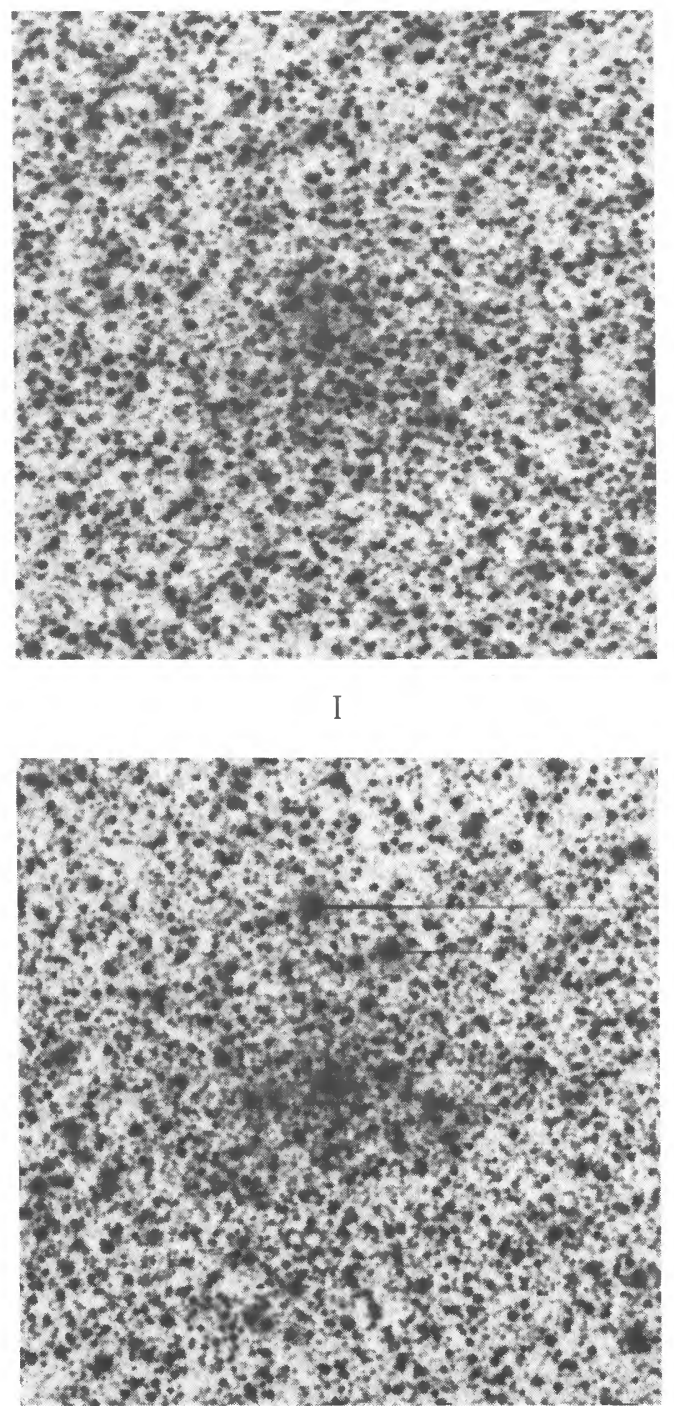

FIG. 1. - CCD images of three new globular clusters, in $R$ (left) and $I$ (right) bandpasses. The fields are $217 \operatorname{arcsec}^{2}$, with north at the top, east to the left. Each of the images was printed by using a histogram equalization technique, and thus the relative contrasts and intensities in these reproductions do not reflect the true colors or surface brightnesses of the clusters.

DJORGOVSKI (see 317 L13) 
TABLE 1

Cluster Coordinates

\begin{tabular}{|c|c|c|c|c|}
\hline Number & $\alpha_{1950}$ & $\delta_{1950}$ & $l$ & $b$ \\
\hline $\begin{array}{l}1 \ldots \ldots \ldots \\
2 \ldots \ldots \ldots \\
3 \ldots \ldots \ldots\end{array}$ & $\begin{array}{lll}17^{\mathrm{h}} & 44^{\mathrm{m}} & 11^{\mathrm{s}} .1 \\
17 & 58 & 40.0 \\
18 & 02 & 59.6\end{array}$ & $\begin{array}{l}-33^{\circ} 02^{\prime} 54^{\prime \prime} \\
-27^{\circ} 49^{\prime} 34^{\prime \prime} \\
-27^{\circ} 46^{\prime} 15^{\prime \prime}\end{array}$ & $\begin{array}{r}356.67 \\
2.76 \\
3.28\end{array}$ & $\begin{array}{l}-2.48 \\
-2.51 \\
-3.31\end{array}$ \\
\hline
\end{tabular}

NoTE.-Positional errors are about 3 " in either coordinate.

"similarity templates" and support the interpretation of the new objects as distant, heavily obscured globular clusters. Visual inspection of the frames suggests that the cluster 3 may have a postcollapse core, whereas the cluster 1 appears to have a flat, King model-like center; the cluster 2 is hard to judge. It is expected that a large fraction of the clusters at small galactocentric radii will have a post-core collapse morphology (Chernoff, Kochanek, and Shapiro 1986; Djorgovski and King 1986).

The coordinates of the clusters, listed in Table 1, were determined from measurements of the SRC Sky Survey films, $I$ print for the cluster 1 , and $R$ prints for the other two. Between 15 and 30 SAO stars were used to establish the coordinate system in each field. The measurements were done with a dual-axis measuring engine at the Center for Astrophysics. The estimated total astrometric errors are $\sim 3^{\prime \prime}$ in each coordinate and are mainly due to the uncertainty of cluster centering (the coordinate transformations contribute only $\sim 0$ ". 5 to the errors).

The clusters are not listed in the Catalogue of Star Clusters and Associations and its supplements (Alter et al. 1961, and references therein), nor any of the published catalogs or lists of globular clusters. A search of published catalogs from other wavelengths failed to produce any cross-identifications: the new clusters are not associated with any of the sources listed in the IRAS Point Source Catalog, Catalog of Infrared Observations (Gezari, Schmitz, and Mead 1984), HEAO-A X-Ray Source Catalog (Wood et al. 1984), or COS B $\gamma$-Ray Catalog (Swanenburg et al. 1981). Cluster 1 is the only one located within a field observed by Einstein ( $H E A O B$ ), viz., IPC field 2543 , and is not detected.

Further analysis of the data must involve a thorough "cleaning" of the foreground stars, color-magnitude array analysis, and photometry, as described by Djorgovski (1987a). In addition to the heavy foreground, the extinction appears to be patchy even across these small-field images. No reliable estimates for the total magnitudes, distance moduli, and extinction can be done from the data in the present form, and such extended analysis for these and some other obscured clusters will be presented in a future paper. The purpose of this Letter is to bring these clusters to the attention of other observers who may want to use them for their own studies.

I wish to thank the staff of Cerro Tololo Inter-American Observatory, and in particular to Jorge Bravo and Mauricio Navarete, for their most valuable help during the observing run. This work was supported in part by Harvard University.

\section{REFERENCES}

Alter, G., Hogg, H. S., Ruprecht, J., and Vanýsek, V. 1961, Bull. Astr. Inst., Czechoslovakia, 12, Appendix 1.

Chernoff, D., Kochanek, C., and Shapiro, S. 1986, Ap. J., 309, 183.

Djorgovski, S. 1987a, in IAU Symposium 126, Globular Cluster Systems in Galaxies, ed. J. Grindlay and A. G. D. Philip (Dordrecht: Reidel), in press.

1987b, in IAU Symposium 126, Globular Cluster Systems in Galaxies, ed. J. Grindlay and A. G. D. Philip (Dordrecht: Reidel), in press.

Djorgovski, S., and King, I. R. 1986, Ap. J. (Letters), 305, L61.

Elson, R., Hut, P., and Inagaki, S. 1987, Ann. Rev. Astr. Ap., 25, in press.

Gezari, D., Schmitz, M., and Mead, J. 1984, Catalog of Infrared Observa-

tions (NASA Reference Publications 1118 and 1119).

Grindlay, J., and Hertz, P. 1981, Ap. J. (Letters), 247, L17.
King, I. R. 1972, Astr. Ap., 19, 166.

Kodaira, K. 1983, IAU Circ., 3846.

Liller, W. 1977, Ap. J. (Letters), 213, L21

Malkan, M., Kleinmann, D., and Apt, J. 1980, Ap. J., 237, 432.

Swanenburg, B., et al. 1981, Ap. J. (Letters), 243, L69.

Terzan, A. 1971 , Astr. Ap., 12, 477. 1971b, Astr. Ap., 15, 336. 1985, ESO Messenger, number 42, p. 4.

Terzan, A., Bernard, A., and Ju, K. H. 1978, C.R. Acad. Sci. Paris (Ser. B), 287, 235 .

Terzan, A., and Ju, K. H. 1980, ESO Messenger, number 2, p. 6.

van den Bergh, S., and Hagen, G. 1975, A.J., 80, 11.

Webbink, R. 1985, in IAU Symposium 113, Dynamics of Star Clusters, ed. J. Goodman and P. Hut (Dordrecht: Reidel), p. 541.

Wood, K., et al. 1984, Ap. J. Suppl, 56, 507.

S. DJoRgovski: Center for Astrophysics, 60 Garden Street, Cambridge, MA 02138 